\title{
How Reliable are Test Numbers for Revealing the COVID-19 Ground Truth and Applying
} Interventions?

\section{Aditya Gopalan* and Himanshu Tyagi}

\begin{abstract}
The number of confirmed cases of COVID-19 is often used as a proxy for the actual number of ground truth COVID-19-infected cases in both public discourse and policy making. However, the number of confirmed cases depends on the testing policy, and it is important to understand how the number of positive cases obtained using different testing policies reveals the unknown ground truth. We develop an agent-based simulation framework in Python that can simulate various testing policies as well as interventions such as lockdown based on them. The interaction between the agents can take into account various communities and mobility patterns. A distinguishing feature of our framework is the presence of another 'flu'-like illness with symptoms similar to COVID-19, that allows us to model the noise in selecting the pool of patients to be tested. We instantiate our model for the city of Bengaluru in India, using census data to distribute agents geographically, and traffic flow mobility data to model long-distance interactions and mixing. We use the simulation framework to compare the performance of three testing policies: Random Symptomatic Testing (RST), Contact Tracing (CT), and a new Location-Based Testing policy (LBT). We observe that if a sufficient fraction of symptomatic patients come out for testing, then RST can capture the ground truth quite closely even with very few daily tests. However, CT consistently captures more positive cases. Interestingly, our new LBT, which is operationally less intensive than CT, gives performance that is comparable with CT. In another direction, we compare the efficacy of these three testing policies in enabling lockdown, and observe that $\mathrm{CT}$ flattens the ground truth curve maximally, followed closely by LBT, and significantly better than RST.
\end{abstract}

\section{Introduction and Summary of Observations}

Beginning March 11, fearing the quick spread of COVID-19, all schools were shut down in Bengaluru, India. Colleges, universities, and cinema halls were soon to follow and were shut down within a week. On Sunday March 22, the Prime Minister of India announced a country-wide "Janta curfew" for a day. Finally, on Tuesday, March 24, the Prime Minister announced a 21-day complete lockdown for the country, which has been extended till May 3rd now. It is interesting to note that there were just 6 confirmed cases and 1 death till March 15 across the state of Karnataka, to which Bengaluru belongs, when the mega-city of Bengaluru was shut down. In fact, till March
1 Indian Institute of Science, Bengaluru 560012, India. *aditya@iisc.ac.in 
24, there were only 517 confirmed cases across India, ${ }^{1}$ a number which may appear small compared to the large $(\approx 1.3$ billion $)$ population of the country. Even the growth rate of the number of cases was not very high. Yet, the policy makers decided to engage a lockdown, perhaps even with popular public support.

Clearly, the perceived number of ground truth cases and its increase rate must have been higher than the actual confirmed cases to facilitate such a drastic measure. But how well does the daily positive test outcome trend reflect the unknown ground truth? Can policy makers use it reliably to implement non-pharmaceutical interventions, such as lockdown, even when the number of daily tests is very low? Such questions become even more important in view of several media articles and expert opinions questioning if India is testing its residents enough $^{20,21}$. That brings out a basic question: How much testing is enough?

In March-April 2020, we initiated a systematic study of this question using a simulation-based framework for testing policies. The findings reported in this paper are based on our study. However, the progression of COVID and the testing policies adopted by the government have evolved rather rapidly over the past few months. We begin our discussion by setting up the larger context, before we return to the discussion on the specific findings of this paper, which not only remain relevant but perhaps have been vindicated over this period.

\subsection{Why do We Test?}

At a high level, a COVID testing policy refers to a sampling algorithm for choosing patients for applying COVID tests. For simplicity of exposition, we do not distinguish between different type of tests and restrict to a single test. Such a test can be characterized mathematically in terms of its sensitivity, the probability that COVID-positive person is correctly identified, and its specificity, the probability that COVID-negative person is correctly identified. The sampling algorithm can depend on observable features such as the personal traits of the individual (for instance, individual's age, gender, comorbidities, presence of symptoms, etc.) or the relation of individual with other individuals (such as whether the individual has contacted another COVID-positive individual).

In our experience, before laying down a testing policy, it is rather important to articulate the

\footnotetext{
${ }^{1}$ These numbers have been taken from ${ }^{2}$.
}

objective the testing policy is supposed to serve. We propose to categorize this objective into three classes:

1. Containment. This is perhaps the foremost objective which the policy makers keep in mind when designing a testing policy. The goal here is to identify and isolate positive cases to contain the spread of infection. This in turn can lead to less burden on medical facilities and lower fatality rates.

2. Discovery. This seems to be the least talkedabout objective of testing, where the goal is to discover new, previously unknown, clusters of infection. An early discovery of a new cluster can allow one to quickly intervene and halt the spread of disease. However, since these new clusters may not be directly connected to previously known cases, this may turn out to be a search problem of formidable complexity.

3. Estimation. The classic motivation for a testing policy is to estimate the number of COVID-positive cases. When forming such an estimate, we need to keep into account the sampling biases and the number of tests used. While theoretically an appealing problem (see, for instance ${ }^{25}$, for related studies), this is a very challenging problem in practice since policy-driven sampling biases are rather difficult to quantify mathematically.

The work reported in this paper mainly focuses on the objective of containment, although we do shed insights on the estimation problem as well. In fact, contact tracing, which has emerged as the backbone of any testing policy, focuses mainly on containment. If one seeks to use the number of positive cases detected using contact tracing to estimate the total number of ground truth cases, it requires a careful modeling of the probability with which contacts are found and selected for testing. This is often a very difficult task, especially in the early stages of an epidemic when the protocols are evolving rapidly and the ground staff is not very systematic. We will elaborate on this issue later in this article.

Also, we remark that to discover new clusters in large populations will require many tests, especially in the initial stages of infection when the statistics are unclear. However, such an objective can be pursued in smaller populations such as those in "containment zones," a smaller 
geographical area cut-off from the remaining parts of the city to contain the spread.

\subsection{The Utility of Modeling and the Simulation Approach}

In our study, we model the progression of disease using probabilistic modeling. This modeling is done at two levels: First, the progression of disease within each individual is modeled using a Markov process; and second, the interaction of different individuals is modeled using a dynamic random graph. In this random graph, we represent individuals by nodes and an edge represents the possibility of transmission of disease between the individuals it connects; see, for instance ${ }^{6,8,13}$.

This model involves several parameters associated with both the within individual progression component and the random graph component. For the first component, the clinical data about each patient can be used to estimate time spent in each state by the patient. Foreseeable challenges here are frequent lapses in data recording and lack of consistent formatting. We have indeed faced such challenges in our own efforts when working with government authorities. Nonetheless, a large amount of clinical data are available, and we believe that tuning the parameters of model for this first part now is quite feasible in practice.

The tuning for second part is more challenging. In the initial stages of the COVID pandemic, our knowledge of interaction graph between people in a geographical region was rather limited. At that stage, a promising strategy was to postulate a probabilistic model for interaction between people based on their features, and estimate the parameters involved using the available interaction data. Such an approach is prescribed in ${ }^{17}$ as well. However, in our experience, such models often turn out to be massively parameterized and many of the parameters cannot be estimated in practice. We note that several Information and Communication Technology (ICT)-driven tools have emerged that provide a lot of information about local interactions in different geographical areas. We believe that the aforementioned random graph models are best suited to merge these data into a systematic interaction model for people.

Once such a model is selected and its parameters are set, a software simulator can be used to emulate the disease progression in an area. This is the approach we follow in the current article, and we will elaborate on this below. At this point, we want to address the concern that has emerged about simulations and predictions for COVID studies over the past few months. As outlined above, the underlying models often have too many parameters to tune, and a common practice is to freeze most of these parameters using reasonable guesses. Only a few of the parameters are tuned based on real data to obtain the desired fits, resulting in a rather ad hoc tuning procedure. As such, the numbers predicted by such modeling and simulation frameworks can deviate from the ground truth in a short span of time (say a month).

However, in the context of our work, we have a different utility for a simulation-based framework. We use our simulation framework to emulate dynamics that capture specific situations that can occur in practice and use it to compare the relative merits of different testing strategies and other interventions that are based on test outputs. We do not tune our parameters to depict the exact recorded numbers, and as such, our findings are not quantitative but qualitative. In this particular use-case, we believe that the role of simulator-based frameworks is unrivalled.

\subsection{Findings of this Work}

We undertake a systematic mathematical modeling and simulation-based study of a variety of testing policies, and compare their efficacy for enabling interventions such as lockdown. Based on our experiments, our main findings can be summarized as follows:

1. If a sufficient fraction of symptomatic population shows up for testing, then testing a small random sample of symptomatic patients can give a good idea of the ground truth trend.

2. Contact tracing (CT), where contacts of a COVID-19 patient are tested, returns a significantly higher number of positive test outcomes than the random symptomatic testing (RST) as above. More importantly, a decision for locking down the population based on CT can help reduce the peak ground truth number of cases ('flatten the curve') much better than RST.

3. Using a location- and mobility patternaware sampling, it is possible to get performance similar to that of CT using operationally less intensive testing procedures. 
We present the precise observations and describe the setup later below, but we quickly note here that we use the derivative of the ground truth curve as an indicator of the ground truth trendindeed, visually it appears to be a good indicator. It is this indicator of ground truth trend that RST reveals well.

Our conclusions are based on agent-based simulation for a population of 100,000 individuals or agents distributed across a realistic synthetic city, interacting based on a realistic mobility pattern. Specifically, we use publicly available census data to distribute the agents across the 198 (urban) wards in the city of Bengaluru. The agents have health states related to COVID-19 and another, generic, 'flu'-like disease condition with similar symptoms, which evolve independently. A susceptible agent (in COVID state S) can get infect when it meets a COVID-19 infected agent (in COVID state I). The agents can interact with other agents in their neighborhood or agents that visit similar locations daily. We instantiate the mobility of agents across the city using mobility data obtained from traffic flows.

This evolution model drives the unknown state, a part of which is observed by testing policies, stored in a separate module in our implementation. A testing policy determines which agents will be subjected to testing and applies a randomized test to the selected agent. The history of test results is stored and is made available to intervention policies, which are stored in yet another module. An intervention policy outputs a control action which modifies the state evolution dynamics. For instance, a lockdown intervention will disable the interaction between the agents. We assume that the borders of the city are closed and there is no interaction with the outside world.

Our overall simulation framework, made available as a Python package at ${ }^{11}$, is flexible and can incorporate any new testing policy or intervention policy. Furthermore, the state evolution model can be easily modified to incorporate more "mixing points" such as buses, malls, etc. In fact, our goal is to incorporate real-time mobility data obtained from digital platforms, as suggested in ${ }^{10}$, to have an updated representation of mixing in the city.

A disclaimer: Our model has not been calibrated to match the actual number of cases in Bengaluru. Neither do our conclusions enjoy rigorous theoretical backing. These results are preliminary and are based on experiments with our simulation framework. They provide, we hope, insight into questions raised above and exhibit the utility of our simulation framework that can used for such studies. This is work in progress, released early to ensure timely dissemination.

\subsection{Related Work}

There is large body of work using mathematical models and simulation to study spread of COVID-19 and facilitate decision-making. A very timely publication was the report ${ }^{9}$ from the COVID-19 response team of Imperial College, London, earlier versions of which raised an alarm about possible worst-case scenarios if COVID19 is allowed to grow unchecked. This in turn is based on a long-line of work from the same group on mathematical modeling and simulation of epidemic spread; see, for instance ${ }^{8}$. Our models of state evolution are similar to these works, but at this point are not as elaborate as these works. More refined models with comorbidity and agedependent evolution have been considered in $^{23}$.

A very elaborate agent-based simulation model for Indian cities has been developed in ${ }^{1}$, 19 . In fact, our simulator is closely related to an initial version of the simulator in ${ }^{1}$; the main difference is the presence of a confounding flu and incorporation of various testing policies, features which have not been considered in these works. In a different direction, the epidemiological framework developed by the INDSCI-SIM group ${ }^{22}$ studies various lockdown scenarios in detail using a differential equationbased simulation model.

While distance-based modeling of interaction of agents is quite popular, with footing in random graph theory, using real mobility and traffic data to model interaction of agents is also gaining prominence in epidemiological studies. In this paper, we have only considered traffic data obtained using surveys, somewhat similar to how data are obtained ${ }^{14}$. A more effective method can be the prescription ${ }^{10}$ where location services and mobile phone usage data are used to obtain real-time daily mobility patterns. Looking ahead, we would like to integrate such data into our framework.

While most of prior work has treated the outcome of tests as the actual ground truth number of COVID-19 cases, very recently, articles ${ }^{17,7}$ have appeared that explicitly study the role of the testing policy. The former has a similar setup as ours, except that the simulation is event driven and uses a more detailed simulation of agent interaction. However, only contact tracing is considered and an ideal situation where there is no other flu with 


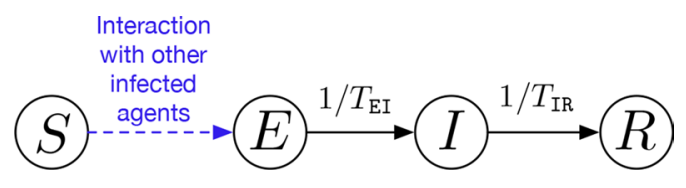

Figure 1: COVID state evolution of each agent.

similar symptoms is assumed. In a way, this framework assumes that one can directly access the ground truth. The article ${ }^{7}$ presents a similar discussion as our results, but the approach appears to be more statistical and not based on explicit epidemic modeling/simulation. We remark that our proposed new testing algorithm uses ideas from multi-armed bandit problems; the paper ${ }^{5}$ proposes an algorithm that uses similar ideas towards achieving a good test allocation strategy.

\subsection{Organization}

The remainder of this paper is organized as follows. We present the details of our modeling and simulation framework in the next section. A comparison of the three testing policies we consider when there is no intervention is given in Sect. 3, and a comparison of their efficacy in enabling interventions in Sect. 4. We conclude with some discussion on policy implications and next steps in the final section.

\section{Simulator Description and Features 2.1 Simulation Model}

We consider an agent-based simulation framework to model the propagation of an epidemic in a city. In our general framework, a city is populated using $n$ agents distributed across fixed "localities" of a city in proportion to their population densities. Each agent $i$ represents a person with attributes such as location associated with it. The health of an agent is captured by its COVID state $C_{i}(t)$ on day $t$. A distinguishing feature of our setup is an additional "flu" state $F_{i}(t)$ which represents the presence of another flu with similar symptoms as COVID-19. This allows us to model the erroneous prescription of COVID-19 test to a person who shows similar symptoms due to presence of another flu, which we believe is essential for a realistic modeling of testing. ${ }^{2}$

\footnotetext{
${ }^{2}$ It is conceivable, however, that the enforcement of public health measures such as recurring lockdowns and face coverings may have rendered other typical respiratory diseases insignificant. Nevertheless, the confounding effects of common symptoms from different respiratory diseases are explicitly factored here.
}

The evolution of $C_{i}(t)$ is determined by two factors: a local evolution model for each agent's state and evolution due to interaction between agents. For local evolution, we use the popular SEIR model for $C_{i}(t)$ where the COVID state of an agent can take values in the set $\{S, E, I, R\}$ representing susceptible $(S)$, exposed $(E)$, infected $(I)$, and recovered $(R)$ conditions, respectively. Note that this model is a simplification of the dynamics used ${ }^{9}$ and combines the several stages such as hospitalization and death into a single state R. The origin of such models in epidemiology can be traced back to the pioneering work of Kermack and McKendrick in 1920s, and they have been used for modeling COVID-19 dynamics ${ }^{10,15,17}$, 23. The collective states $C(t)=\left\{C_{i}(t), 1 \leq i \leq n\right\}$, $1 \leq t \leq T$, form a discrete-time Markov chain, where $C_{i}(t)$ on day $t$ changes to the state $C_{i}(t+1)$ on day $t+1$ according to a pre-specified probability transition structure, which is depicted in Fig. 1. Specifically, each agent changes its state from $\mathrm{E}$ to I to $\mathrm{R}$ independently of all the other agents. The transition probabilities in Fig. 1 are set to parameters $1 / T_{s s^{\prime}}$, where $T$ is the average transition time between states $s$ and $s^{\prime}$. Although the values chosen for our simulations are only approximations, such average times have been studied and reported extensively in literature, and the most up-to-date estimates can be used in the model by the interested practitioner.

Note that an agent makes a transition from $S$ to $E$ based on its interaction with other agents. When an agent in state $I$ meets another agent in state $S$, the latter agent gets infected with a pre-specified probability $p$. To model the meeting of agents, we include two components: The first is a "neighborhood" component where each agent meets a set of randomly chosen agents from the same neighborhood. In addition, each agent meets a fixed set of agents from its neighborhood, generated randomly at start and then fixed throughout the simulation. Note that, for an agent, its neighborhood need not coincide with its locality and can include a set of close-by localities. In our simulation, we have defined a neighborhood as a set of localities touching (geographically) a given locality.

The second component represents interaction with agents from different localities, not necessarily the neighboring ones. Here we propose to use data about mobility in the city. Here, too, an agent visiting a location on a day interacts with a set of randomly chosen agents generated afresh everyday as well as fixed set of agents set upfront.

One final component of our model is the evolution of the "flu" state $F_{i}(t)$. We remark that the 
inclusion of this state is only to model the noise in applying a testing policy. Thus, we use a simple, intrinsic, SIS model for $F_{i}(t)$ where it is evolves as a Markov chain independently for each $i$ and taking values in $\{S, I\}$ with transition probabilities depicted in Fig. 2. Note that an important distinction between the COVID and Flu models is that in the former, the process "terminates" once the state $R$ is reached since we assume that a person who has been infected with COVID-19 once cannot be infected again. However, a person keeps on shifting from $S$ to $I$ Flu states indefinitely-this is because the "flu" state is an proxy for the individual contracting any illness with similar symptoms as COVID-19. ${ }^{3}$ As we shall elaborate later, any testing policy that makes a pool of symptomatic patients will treat an agent with $C_{i}(t)=I$ and $F_{i}(t)=I$ identically (if all their other features such as locality match).

This template for an interaction model is very generic and can incorporate various movements across the city in form of origin-destination flow data. Even bus routes can be included by considering them as locations. However, we have only implemented a restricted form of this interaction in the first version of our simulator used for experiments in this paper. Specifically, the number of fixed and randomly selected agents met in the neighborhood and the locality visited are fixed to be the same for all the agents. Furthermore, only one visited location is set per agent, as a part of its feature matrix. However, we remark that it is easy to extend our model to include multiple locations (such as workplace, bus-used, etc.).

Before specifying the parameters used for the results of this paper in the next section, we remark that our framework requires mobility data to be fed in the form of an origin-destination (OD) matrix. Such a matrix has number of rows $N_{r}$ as the number of localities and the number of columns $N_{c}$ representing the locality or community visited daily. The $(i, j)$-th entry of this matrix represents the probability with which a person from locality $i$ goes to locality $j$, whereby the $i$ th row constitutes a probability vector that sums to one. We use this vector to generate the locality visited by each agent in locality $i$, independent of other agents. Note that we can have multiple such OD matrices, one for each community, and each person can be a member of one community each for every matrix available in the model. In its current form, our implementation allows the membership to depend only on the location of the agents. Furthermore, agents have not been endowed with other potentially relevant features such as age and comorbidity which determine COVID state

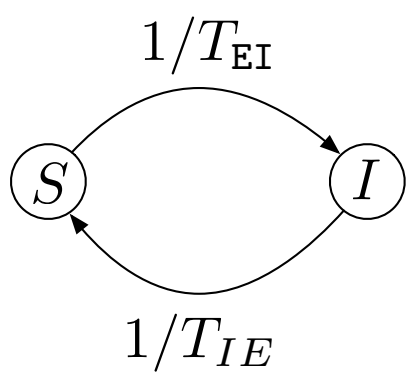

Figure 2: Flu state evolution of each agent.

evolution in practice. We plan to include these enhancements in a subsequent version.

We close this section by noting that the state evolution of our model is defined in the file evolution.py of our implementation. Also, we point out that the state of the city is captured by a pandas data frame City Population, abbreviated as $\mathrm{CP}$, with $N$ rows and columns corresponding to various features of each agent.

A caution: Our implementation is designed to use multiple CPU cores in parallel, but the specific dynamics change when number of cores are increased. In particular, increasing the number of cores used changes the number of state transitions happening in parallel, reducing the overall growth rate. Throughout this paper, we have set the number of cores to 8 .

\subsection{Parameters Used for Simulation}

In this paper, we instantiate the general framework described above for the city of Bengaluru in India. For simplicity, we only consider an SIR model for COVID state where the $E$ state is skipped by setting $T_{E I}=1$. We consider the 198 urban wards of Bengaluru that come under the city municipal corporation (Bruhat Bengaluru Mahanagara Palike, BBMP) and use the census data (converted to a .geojson file using data from $^{3,4}$ ) to populate $N=100,000$ agents across the city; see Fig. 3 for depiction of population densities.

For modeling mobility across the city, we use data on vehicle mobility across Bengaluru acquired from the Centre for Infrastructure, Sustainable Transportation and Urban Planning (CiSTUP), Indian Institute of Science, Bangalore. This is similar in spirit to the prescription ${ }^{10}$, but instead of dynamic digital data, we use static data

\footnotetext{
${ }^{3}$ It is possible that the time scale of natural immunity to influenza-like illnesses is much longer than the model's time horizon, in which case the SIS model for "flu" will not actually cycle through more than one infection during the horizon.
} 


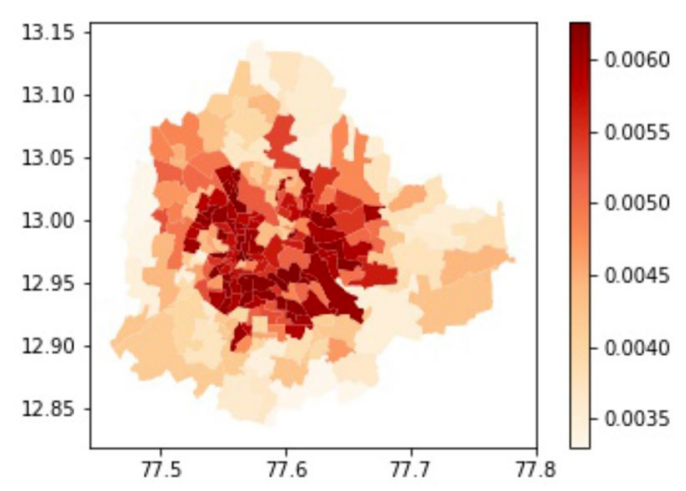

Figure 3: Ward-wise population density of Bengaluru.

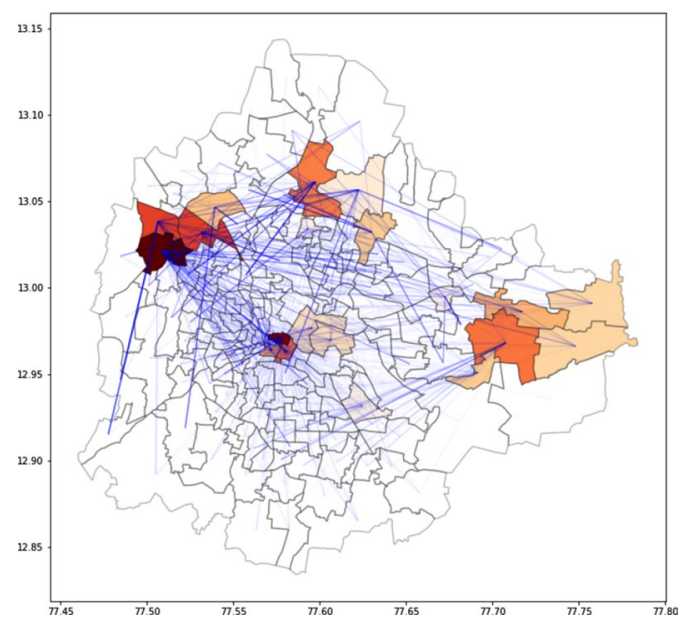

Figure 4: The top 20 wards with highest car traffic inflow in Bengaluru. The red component in the color of each ward is proportional to the fraction of inflow traffic for the ward and the opaqueness of blue lines indicate the flow between the wards it joins.

obtained using surveys similar to ${ }^{14,15}$. These data include OD matrices for vehicles of different categories across Bengaluru which was obtained by conducting household surveys across the city. We have only used the data for car traffic in our interaction model. Furthermore, it includes daily bus ticket sales obtained from BBMP; however, in this paper, we have not used the bus ticket sales data. To reduce computational load, we restrict the OD matrix to the 20 destination locations seeing the highest inflow, depicted in Fig. 4. Note that we also have a fictitious destination 21 representing an agent not visiting any of these 20 destination. With the interaction model set, we now list the values set for various parameters of our model described in the previous section:

\begin{tabular}{|c|c|}
\hline Parameter & Value \\
\hline COVID infection rate $p$ & 0.1 \\
\hline Average time from $E$ to / for COVID state $T_{E I}$ & 1 \\
\hline Average time from I to $R$ for COVID state $T_{\mathbb{R}}$ & 8 \\
\hline Average time from $S$ to $/$ for Flu state $T_{S /}$ & 50 \\
\hline Average time from / to $S$ for Flu state $T_{\text {iS }}$ & 8 \\
\hline $\begin{array}{l}\text { Number of randomly selected people each person } \\
\text { meets in its neighborhood }\end{array}$ & 1 \\
\hline $\begin{array}{l}\text { Number of fixed people each person meets in its } \\
\text { neighborhood }\end{array}$ & 5 \\
\hline $\begin{array}{l}\text { Number of randomly selected people each person } \\
\text { meets at its workplace locality }\end{array}$ & 2 \\
\hline $\begin{array}{l}\text { Number of fixed people each person meets at its } \\
\text { workplace locality }\end{array}$ & 10 \\
\hline
\end{tabular}

We remark that while our focus in this paper is not on careful calibration of the model, we have used reasonable parameter values based on results ${ }^{12,16}$. We execute each simulation for $1,00,000$ people for a duration of $T=100$ days. We mention in passing that we can easily incorporate bus data as well as movement data collected from location traces as suggested ${ }^{10}$ in our framework; we will do this in subsequent versions of our implementation.

\subsection{The testing policy framework}

A strength of our framework is its ability to incorporate testing policies. Our simulator takes as input a function testingPolicy described in the file tests.py. The history of testing of entire population is stored in an $(N \times T)$ matrix TestingHistory, which stores 0 as the default value for each entry and updates the $(i, t)$-th entry to 1 or -1 , respectively, if agent $i$ is tested on day $t$ and the outcome is positive or negative.

We can admit any testing policy that selects a pool of candidates to be tested and applies a common test function test() to each individual in the pool.The test function is defined to have 0 probability of a false positive (this is in line with observed characteristics of the standard Reverse Transcriptase Polymerase Chain Reaction (RTPCR) test for COVID-19 $9^{27}$ ), but a nonzero probability of false negative can be set. For simplicity, we have set the probability of false negative to 0 in our simulations. ${ }^{4}$

In selecting the pool of candidates, a testing policy can only use observable features such as locality of individual, the workplace visited, and whether an agent is symptomatic. Note that a symptomatic agent can have either Flu state I or COVID state I, but a testing policy (obviously 
so) cannot use the actual COVID or Flu state of a person.

We have implemented and simulated three testing policies. Each policy selects a fixed number $N_{\text {test }}$ of symptomatic agents at each time step (day) to apply tests. The following policies are simulated:

1. Random Symptomatic Testing (RST): The daily pool of agents to be tested is selected randomly (amongst people who are symptomatic).

2. Contact Tracing (CT): The daily pool is selected randomly from the set of all symptomatic among the neighborhood contacts and the workplace contacts of all the patients. If this set is of smaller cardinality than $N_{\text {test }}$, which happens often, we enhance it with symptomatic from across locations.

3. Location-Based Testing (LBT): This policy is a bit involved and will be described in detail in Sect. 3. At a high level, this policy gives priority for testing to symptomatic agents belonging to localities and workplaces with higher infection.

A few remarks are in order. First, these policies are enabled by maintaining a list of fixed contacts in the neighborhood and the workplace, which can be identified for CT. We assume that the randomly selected daily contacts that an agent interacts with cannot be traced, as is the situation in practice.

Second, we mention that CT is an "infraheavy" testing policy requiring operational support to trace contacts and test them. In contrast, RST and LBT are algorithms that do not require operational support but require a careful design of policy mechanisms to ensure prescribed sampling.

Finally, we briefly outline the connection between testing policies in practice and the ones we have implemented in our simulation framework. All the testing policies described above start with a list of symptomatic agents. In practice, such a list emerges when symptomatic patients reach out to their medical provider. An effective information campaign by the government can ensure that patients with symptoms matching those seen in COVID-19 come out for testing. Nonetheless, the exact percentage of symptomatic patients that come out can vary with localities, since each locality differs in income levels and availability of medical facilities. To model this, we incorporate "under-reporting" in our framework which can be represented by a locality wise reporting probability vector. Further, our tests sample randomly from the pool of symptomatic patients. In practice, this sampling is implemented by medical professionals who recommend a subset of symptomatic patients for testing. We believe that all the policies we have simulated can be (and, for some, have been) converted to an implementable form on the ground at least in the Indian context, e.g., the containment plan of the Ministry of Health and Family Welfare, Govt. of India, already lays down "hotspot detection' as part of a testing/intervention strategy ${ }^{18}$.

\subsection{The Interventions Framework}

Our framework can simulate evolution under interventions. A policy intervention such as a city-wide lockdown is implemented as modifying the interactions between different agents in the simulation. The intervention policy is specified as an input in the form of Python function interventionPolicy which is accessed everyday (for every iteration) to produce a list of interventions. When updating the state of each agent (using the function updatestate specified in evolution.py), the list of interventions enabled on each day is used to decide which interactions will be allowed for the agent. This is done by accessing the function InterventionRule, which interprets the impact of intervention for state evolution. In particular, we have implemented and simulated the following interventions in the module interventions . py:

1. Quarantine (Python function InterventionQuarantine): Each agent that is tested positive for COVID-19 on day $t$, along with all the agents on its list of neighborhood and workplace contacts, are placed on quarantine for a period of 10 days starting from day $t+1$. Namely, the agent is not allowed to interact with any other agent during this quarantine period.

2. Indefinite lockdown (Python function InterventionLockdown): All agents are not allowed to interact with any other agent once a particular trend is detected in the positive test count.

3. Fixed duration lockdown (Python function InterventionLockdownFixed): All agents are not allowed to interact with any other agent for a fixed period of time once a particular trend is detected in the positive test count.

${ }^{4}$ The noise in tests appear because of the presence of another flu with similar symptoms as COVID-19. 
In the last two lockdowns, the rule to start a lockdown has not explicitly been mentioned. We will elaborate on this later, but roughly, a lockdown is triggered once the slope of positive COVID-19 tests crosses a threshold. Thus, the efficacy of an intervention policy is tied intrinsically to the testing policy used. As such the intervention policy function is given access to the overall population state $\mathrm{CP}$, the history of interventions imposed, and the testing history matrix. If an additional state is required for implementing a policy_such as quarantine requires us to store the quarantine state of each agent-it is included as a part of CP.

In summary, our simulation framework separates state evolution functions (in evolution.py), testing policy (in tests.py), and intervention policy (in interventions.py). A new test or a new intervention can easily be incorporated by maintaining the input-output structure.

\section{Testing Strategies and Performance Comparison}

This section details the testing strategies that we have explored using the simulation framework of Sect. 2, and compares their performance. For this section, no intervention is performed based on the test outcomes. The impact of tying interventions to test outcomes is discussed in Sect. 4.

\subsection{Testing Strategies}

At a high level, a test strategy is expressed as a test selection rule, applied in each time step (day) of the simulation, that maps the current population, together with the past testing history, to a subset of individuals that are subsequently tested for COVID-19 infection. This map cannot depend on the actual health states of the individuals (e.g., their COVID state), but can rely on only their observable attributes, such as whether they display symptoms of illness or whether they reside in a specific ward or wards. The map can also be randomized to reflect random sampling from certain locations without necessarily relying on the onset of symptoms. The general pseudocode of a test selection rule appears in Algorithm 1.

All individuals selected by a test selection rule are assumed to undergo medical testing (e.g., RT-PCR testing) represented by the individual test subroutine Algorithm 2 (defined as the function test in tests.py), which models false negatives arising in the testing process at an assumed rate $r \in[0,1]$.

In the sequel, the testing strategies we describe are specifications of test selection rules, assuming access to a standard individual testing subroutine.

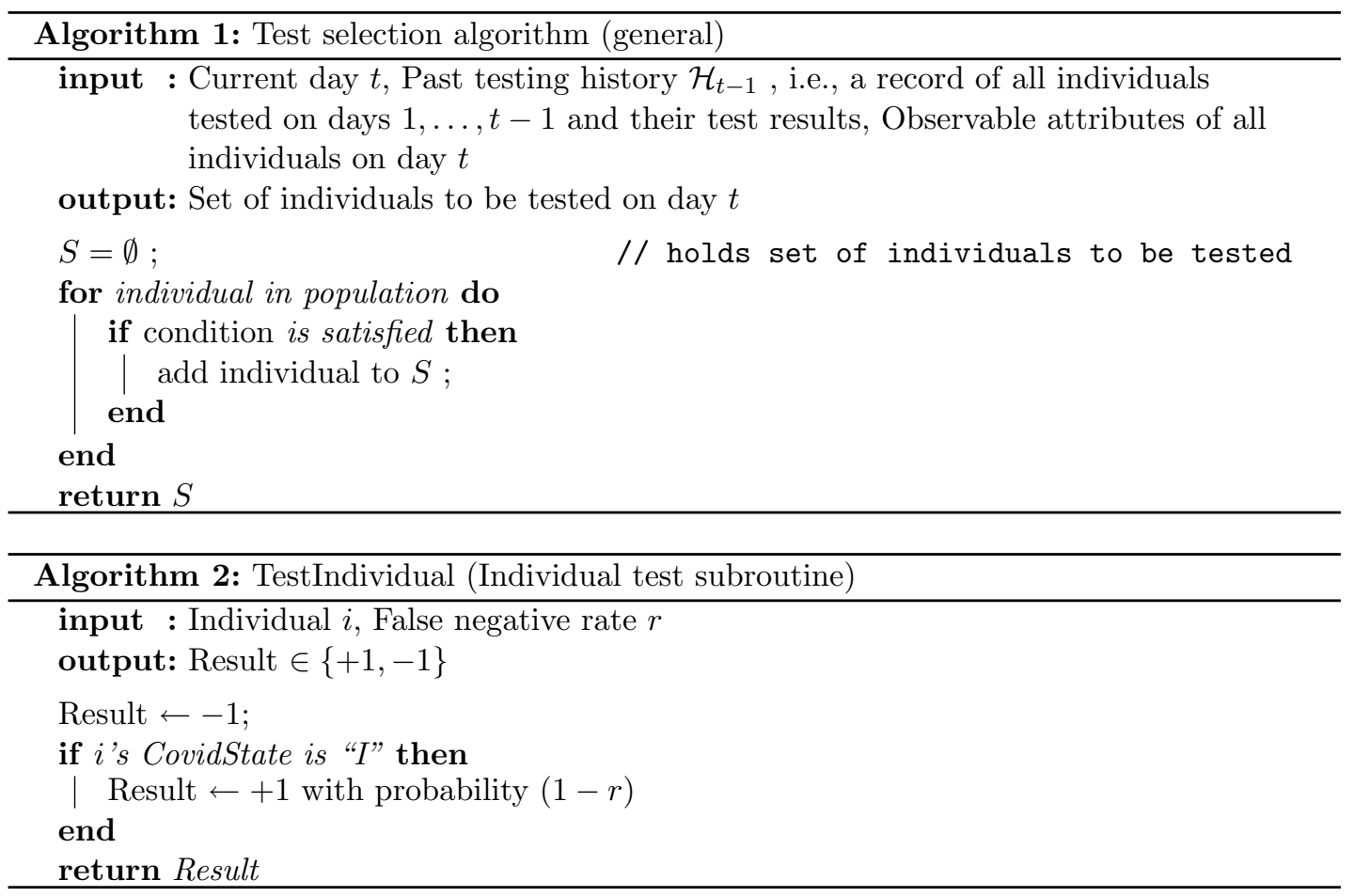




\subsubsection{Random Symptomatic Testing}

The first, and simplest, testing strategy we consider is Random Symptomatic Testing (RST). This strategy (pseudocode in Algorithm 3) looks at the pool of people who display symptoms typical of the disease, and randomly samples as many of them as possible to fill up a predefined budget. This is considered a baseline testing strategy under budget constraints in the experiments reported here.

\subsubsection{Location-Based Testing}

Location-Based Testing (LBT) is a testing rule that is designed to favor individuals who are 'close' to the currently known footprint of the COVID-19 infection. Closeness here is assumed to be high if (a) the individual's locality contains many individuals known to have tested +ve in the past, or (b) many individuals who have tested +ve in the past are associated with the individual's visit place.

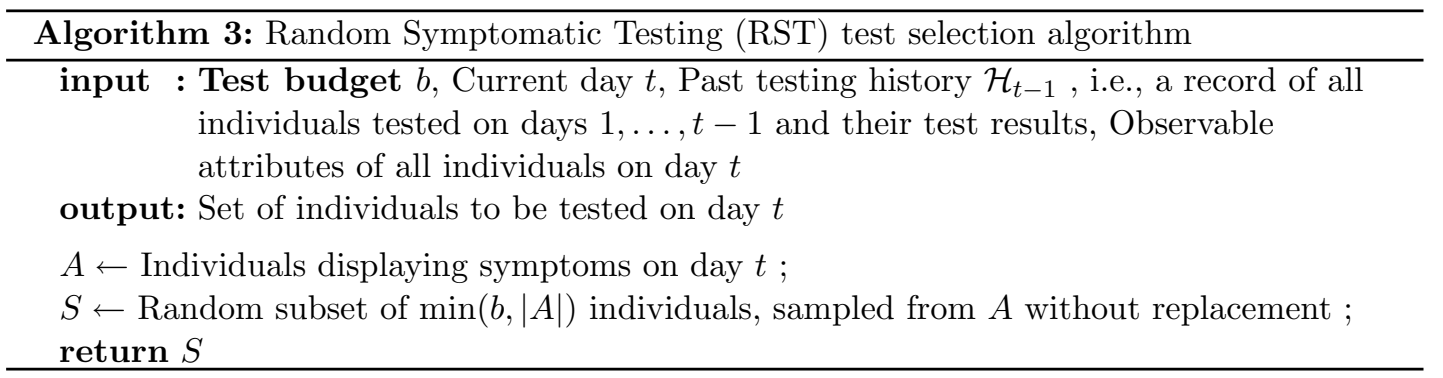

\subsubsection{Contact Tracing}

Contact Tracing (CT) is a testing strategy in which symptomatic contacts of individuals that have tested positive in the recent past ${ }^{5}$ are tested at the highest priority. If any more tests are available, then as many (randomly chosen) individuals as possible that are presently exhibiting symptoms are chosen for testing. Pseudocode for the CT test selection strategy appears in Algorithm 4. Note that in practice even the nonsymptomatic contacts are often tested, which is what should be done in a simulation with the SEIR model. But since we have disabled the ' $E$ ' (exposed) state for simplicity, we only test symptomatic contacts.
The LBT selection rule (Algorithm 5) essentially computes a closeness or risk score of each person who reports symptoms, and prioritizes individuals for testing depending on their risk scores. The risk score of a person can be thought of as a crude proxy for the posterior probability of that person being infected with COVID-19 on a given day, given all the observed history of tests.

For our implementation, we define the score of an individual $i$ on day $t$ as a weighted sum of the scores of its residence locality and its visit place. The score of a locality is an exponentially weighted average of the number of +ve tested individuals associated to it in the past, e.g., an individual who tested positive from the locality

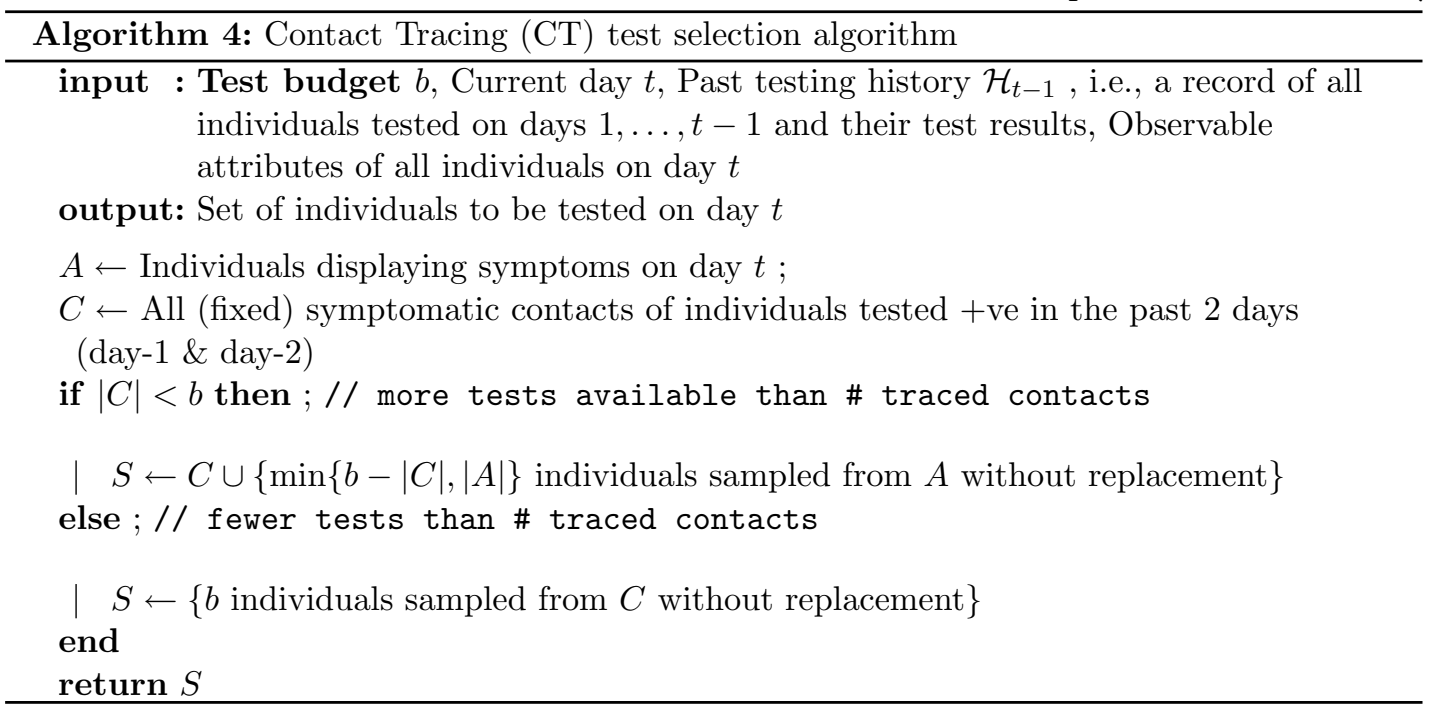

\footnotetext{
${ }^{5}$ In our implementation of CT, the recent past is taken to be the past 2 days.
} 


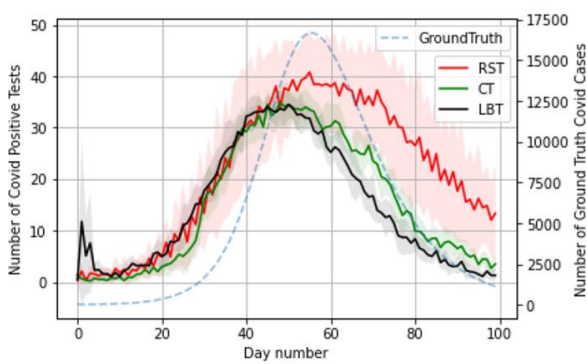

(a) Daily number of tests.

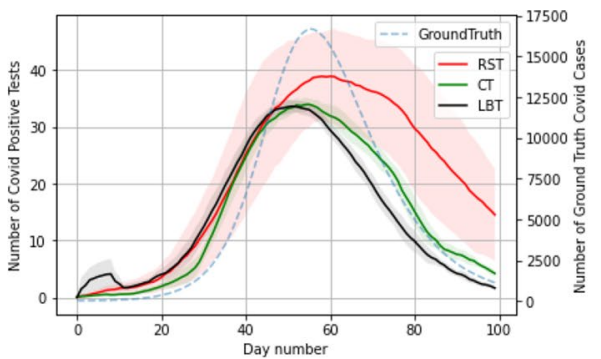

(b) Daily number of tests smoothened by taking an average over a window of 8 days.

Figure 5: Comparative test performance with clustered seeding and without intervention, for a time period of 100 days and with a testing budget of 50 tests/day. Results are averaged across 10 runs and error bars represent 1 standard deviation.

$\Delta$ days ago contributes $\alpha_{\text {loc }}(1+\epsilon)^{\Delta-1}$ to that locality's score where $\alpha_{\text {loc }}>0$ is an adjustable parameter. The score of a visiting place is defined analogously. Pseudocode for the LBT selection algorithm appears in Algorithms 5 and 6.

\section{Remark}

We have not modeled the fact that both the conduct of tests and their reporting can suffer delays. For the interested experimenter, this can be incorporated
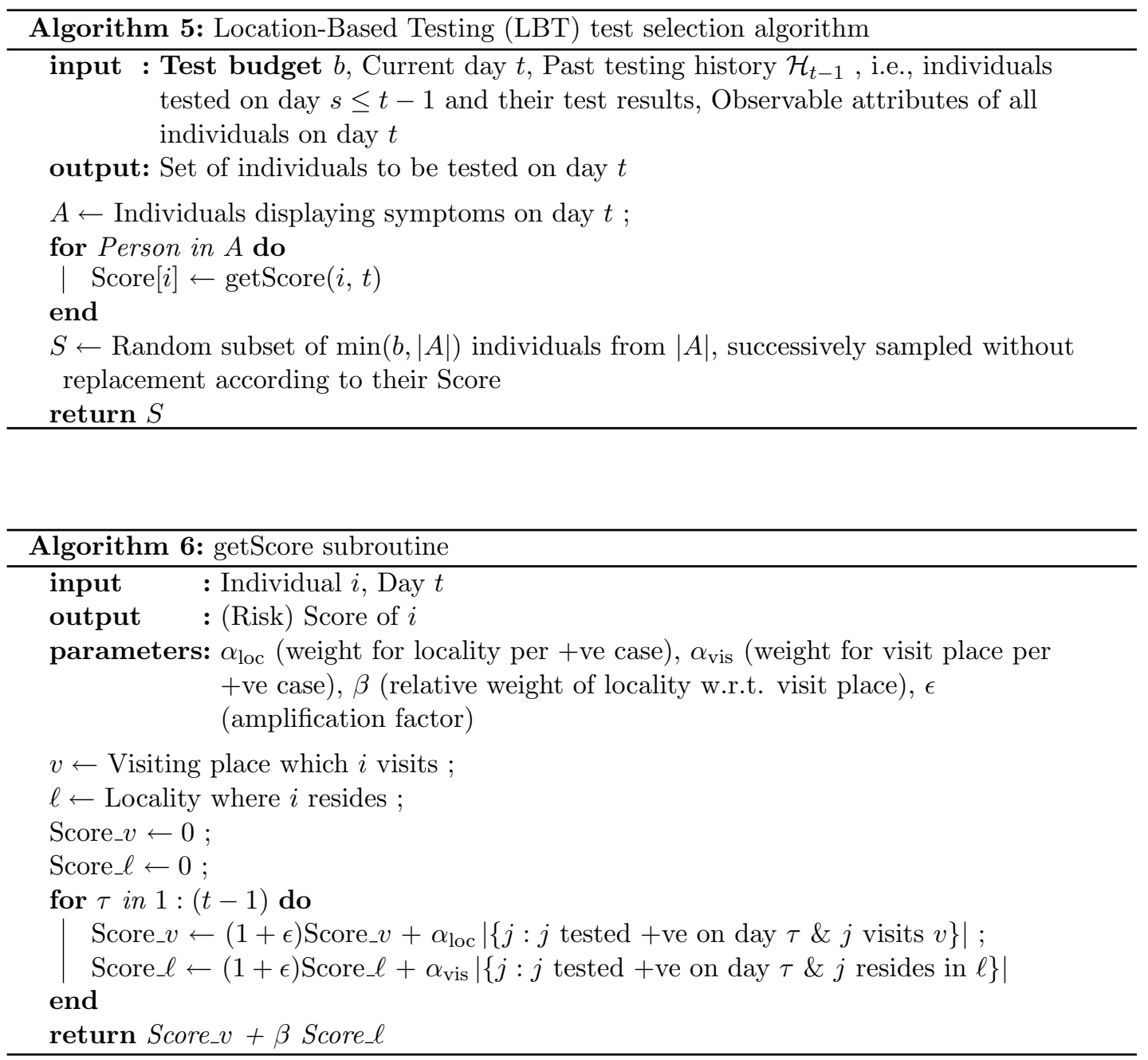


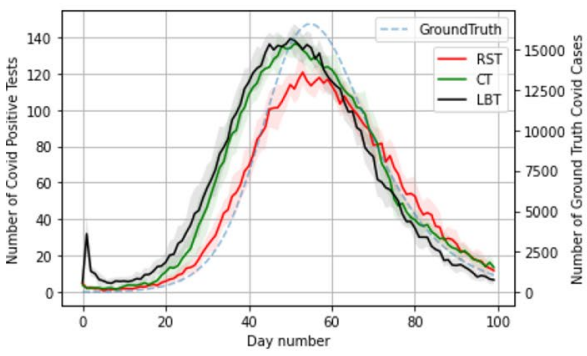

(a) Daily number of tests.

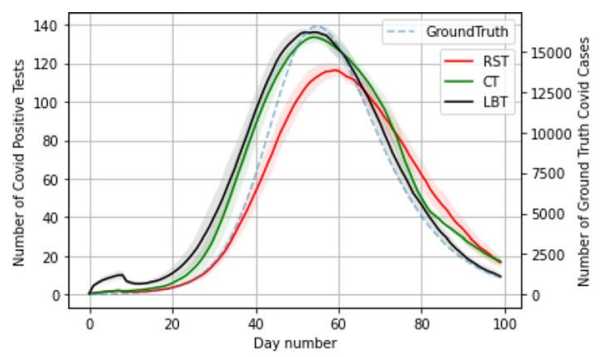

(b) Daily number of tests smoothened by taking an average over a window of 8 days.

Figure 6: Comparative test performance with clustered seeding and without intervention, for a time period of 100 days and with a testing budget of 200 tests/day. Results are averaged across 10 runs and error bars represent 1 standard deviation.

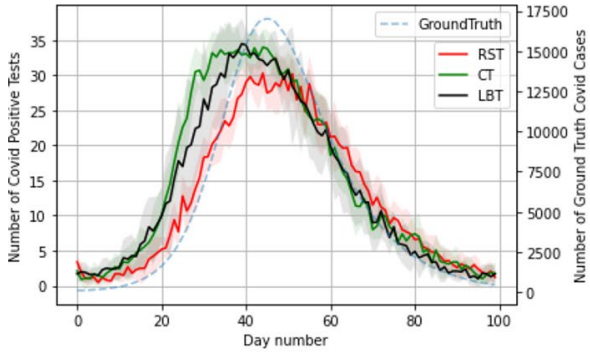

(a) Daily number of tests.

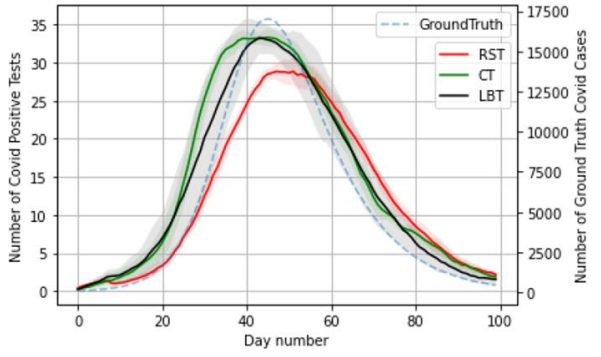

(b) Daily number of tests smoothened by taking an average over a window of 8 days.

Figure 7: Comparative test performance with uniform seeding and without intervention, for a time period of 100 days and with a testing budget of 50 tests/day. Results are averaged across 10 runs and error bars represent 1 standard deviation.

easily by having the result of the TestIndividual subroutine (Algorithm 2) available to the parent test selection routine after a suitable delay.

\subsection{Numerical Results and Discussion}

We present and contrast here the numerical performance of the 3 testing strategies described previously-Random Symptomatic Testing (RST), Contact Tracing (CT) and Location-Based Testing (LBT). We run our experiments using the parameter settings of Sect. 2.2, without any nontrivial interventions enabled for individuals who test positive for COVID-19.

\subsubsection{Test Performance with Clustered Seeding} Clustered seeding simulates the initial condition where all the initial COVID-19 cases are spatially localized. In our experiments, we initialize 50 COVID-19-infected individuals (out of a population of $100 \mathrm{~K}$ ) in a single locality (ward number
120 , the 'Cottonpete ward') of the city of Bengaluru and let the outbreak evolve from there.

Figure 5 depicts the mean daily test score (number of tests with positive results) evolution with time for 10 independent simulation runs, along with the corresponding 1-standard deviation ranges shaded in lighter color, for a daily budget of 50 tests/day. The ground truth number of cases per day is also plotted in the background. It is interesting to note that (a) RST shows high variance in reporting compared to the more biased CT and LBT, (b) while all 3 tests are equally accurate in capturing the trend of the ground truth in the phase leading up to the peak of actual case count, their performance after the peak is reached is quite different-CT and

\footnotetext{
${ }^{6}$ We have not formally defined the notion of "trend," but a good proxy to keep in mind is the derivative of the ground truth curve, which seems to be well approximated by the derivative of the positive tests curve. This notion of approximation is well studied in nonparametric statistics, and can be used for a theoretical treatment of COVID-19 ground truth estimation.
} 


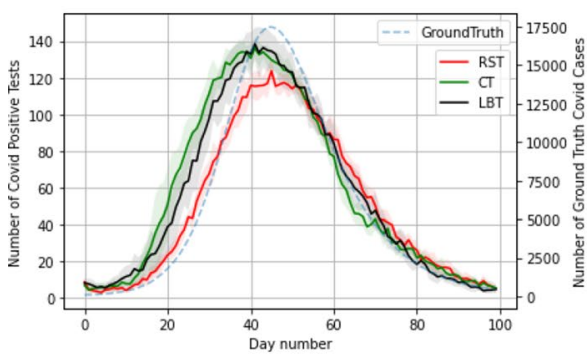

(a) Daily number of tests.

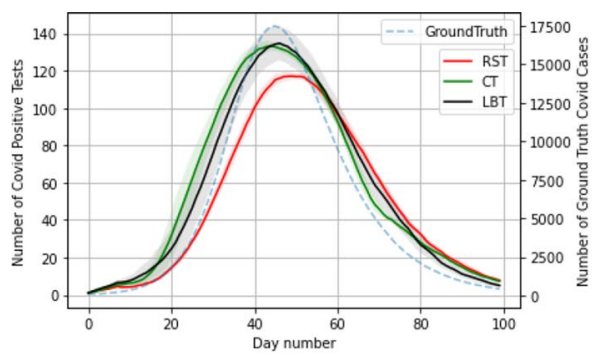

(b) Daily number of tests smoothened by taking an average over a window of 8 days.

Figure 8: Comparative test performance with uniform seeding and without intervention, for a time period of 100 days and with a testing budget of 200 tests/day. Results are averaged across 10 runs and error bars represent 1 standard deviation.

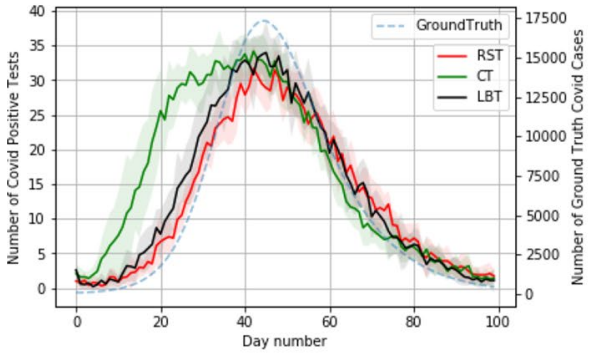

(a) Daily number of tests.

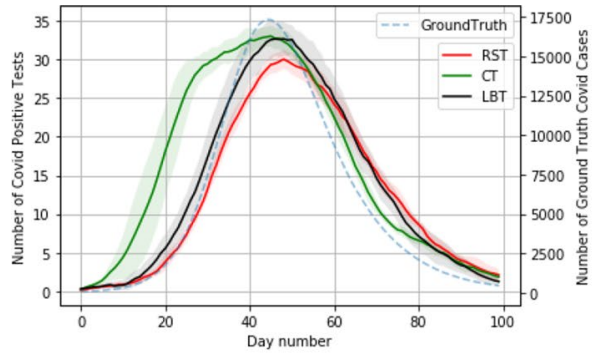

(b) Daily number of tests smoothened by taking an average over a window of 8 days.

Figure 9: Comparative test performance with uniform seeding and without intervention, for a time period of 100 days, a testing budget of 50 tests/day, and $10 \%$ reporting of symptoms. Results are averaged across 10 runs and error bars represent 1 standard deviation.

LBT tend to fall earlier than RST. ${ }^{6}$ Also interesting is the short-lived peak in the curve for LBT early on-this is due to the test aggressively prioritizing testing from the affected seed locality. A smoothed version of the test results, plotted alongside, shows that smoothing can make the outputs of CT and LBT capture the rise-fall trend of the ground truth in a better fashion. Note that we have chosen a smoothing window of 8 days since the average time from COVID state ' $T$ ' to COVID state ' $R$ ' in our model is 8 days. Presumably, the gains due to smoothing can be attributed to this fact-a patient tested positive 8 days ago is likely to remain positive till the current day.

We also compare the tests with an enhanced $(4 \times)$ budget of 200 tests/day (Fig. 6). The extra budget appears to put to good use by the 'smart' tests CT and LBT, whose test numbers outstrip those of RST by a significant margin in the lead up to the peak. The faithfulness to the actual ground truth signal also appears to be much better for CT and RST here.

\subsubsection{Test Performance with Uniform Seeding}

Figure 7 shows the results of applying the 3 tests with an initial seeding that is uniform across localities (city wards). Specifically, each of the (approx.) 200 localities in the simulation model hosts an independent Binomial $(5,0.1)$ number of COVID-19-infected seeds at start, resulting in about 100 seeds in the overall population $(100 \mathrm{~K})$. Figure 8 plots the same metrics but for tests with an enhanced testing budget (200 per day).

The results show a clear advantage of the more advanced tests (CT, LBT) over the RST baseline, in the period leading up to the peak of ground truth COVID-19 cases. This potentially brings out the value of relying on predictive biased sampling (over and above the symptomatic sampling by RST) to detect a higher number of cases in the initial stages of the outbreak. We will see later (Sect. 4) that this confers a significant advantage in terms of timing when 


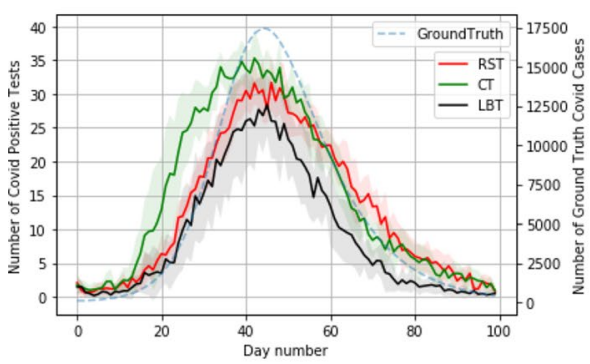

(a) Daily number of tests.

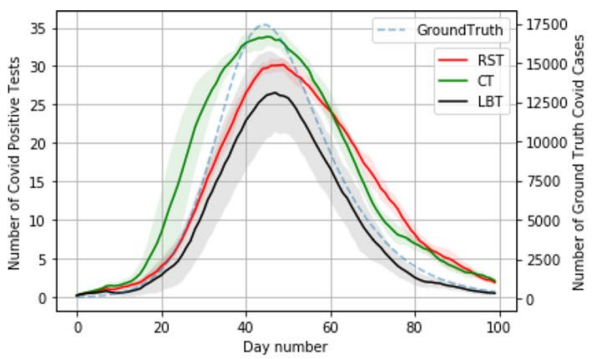

(b) Daily number of tests smoothened by taking an average over a window of 8 days.

Figure 10: Comparative test performance with uniform seeding and without intervention, for a time period of 100 days, a testing budget of 50 tests/day, and non-uniform reporting of symptoms. Results are averaged across 10 runs and error bars represent 1 standard deviation.

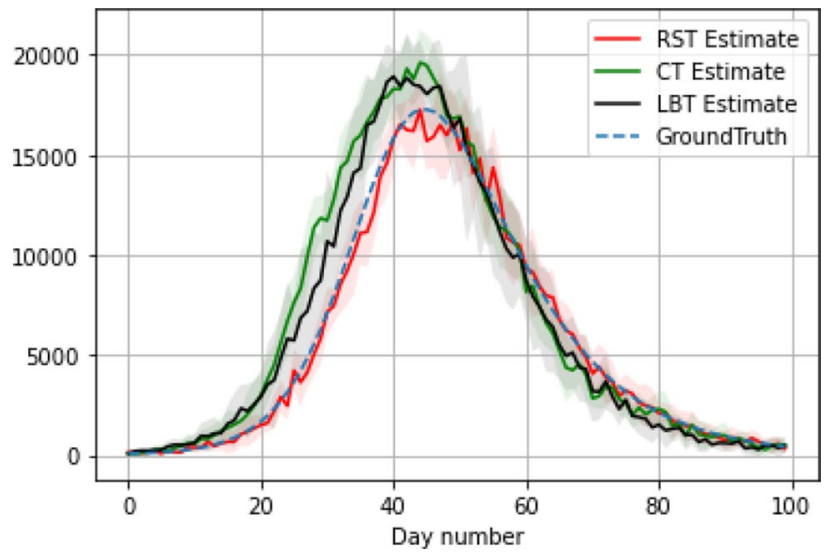

Figure 11: Estimated no. of COVID-19 cases computed using numbers of symptomatic (at 100\% reporting) and positive case counts. The simulation was carried out with uniform seeding and no intervention and a testing budget of 50 tests/day.

the results of the former tests are used to implement public (large-scale) lockdowns.

\subsubsection{Test Performance with Under-Reporting of Symptoms}

We also examine what happens when individuals under-report symptoms when ill. This has the effect, in our simulation, of reducing the initial pool of symptomatic individuals which are input to the test selection strategy.

Uniform under-reporting. Figure 9 shows the test performance curves when each individual who is infected (either with COVID-19 or "flu") is assumed to report as symptomatic with probability 0.1 independently (in the previous sections, this was assumed to occur with probability 1$)$. Though the $10 \times$ under-reporting does not significantly affect the way in which all 3 tests capture the rise and fall in ground truth cases, contact tracing emerges as the most informative detector of cases in the lead up to the peak.

Non-uniform under-reporting. Figure 10 depicts the tests operating in a scenario where individuals report in a non-uniform manner whether they are symptomatic and hence to be considered for testing. Specifically, individuals from about $1 / 3$ rd of the localities (wards) in the city (selected at random) report symptoms at rate $5 \%$ while the rest report at rate 100\%. The initial seeding for COVID-19 cases is uniform across the localities as before. Contact tracing is seen to be robust to the rather skewed under-reporting enforced here, presumably due to its more accurate predictions of infection targets due to a richer local signal. 

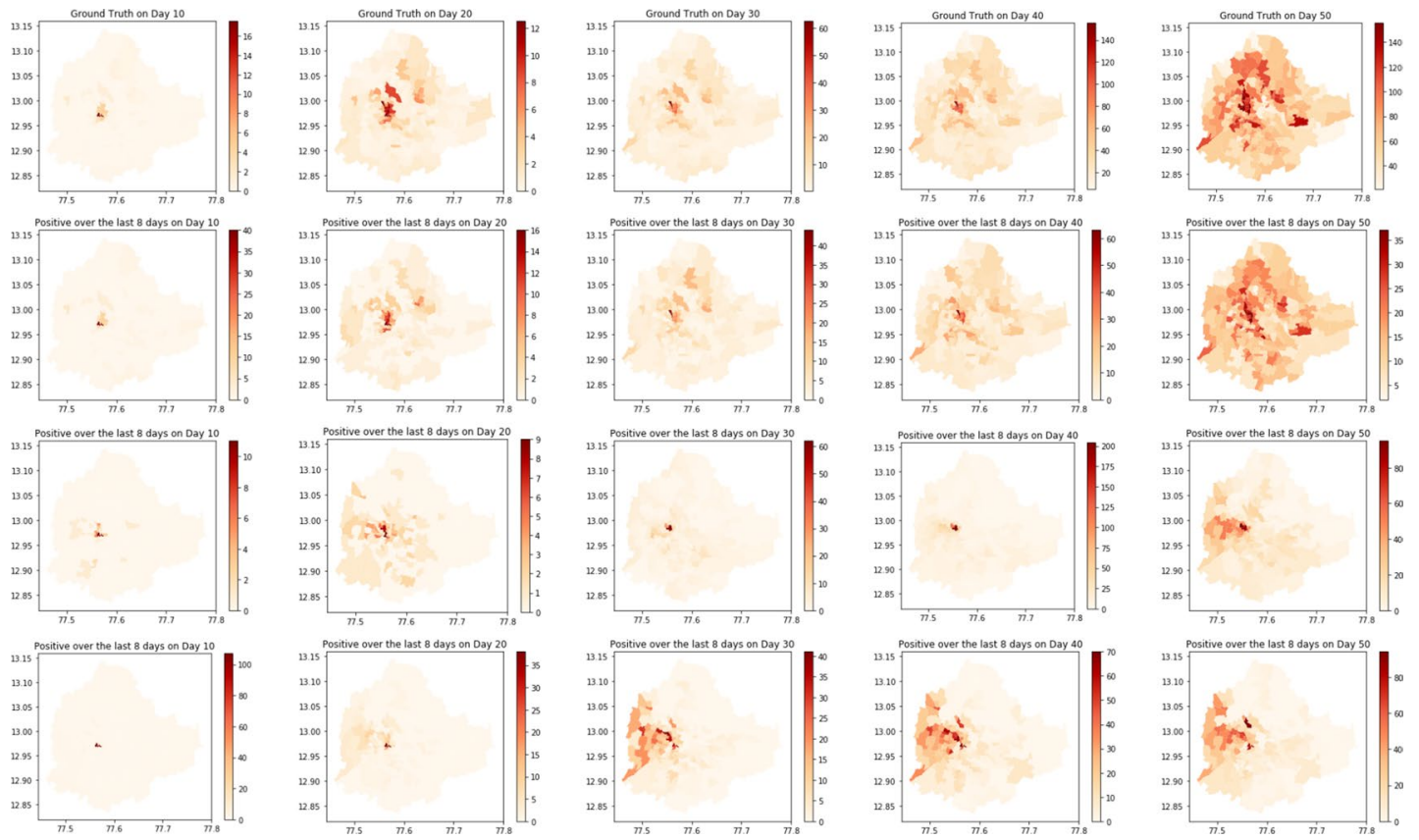

Figure 12: Geo plots illustrating the evolution of the ground truth and positive cases across the city and over time, with uniform seeding of COVID-19 cases. The $X$-axis represents time, with each successive column denoting an increment of 10 days from the previous column (or day 0 for the first column). The first row represents the heat map for simulated ground truth COVID-19 cases by locality in Bengaluru. The second, third and fourth rows represent the heat maps for COVID-19-positive cases detected per locality by the Randomized Symptomatic Testing (RST), Contact Tracing (CT) and Location-based Testing (LBT) test selection algorithms, respectively, for the past 8 days.

\subsubsection{Estimating the Ground Truth Number of Infections}

We explore the question of how well the test results, along with a record of the number of symptomatic individuals considered for testing, can be used to estimate the ground truth number of COVID-19 infections at any point in time. To this end, Fig. 11 plots, for each day, an estimate of the ground truth computed as: the number of symptomatic patients $\times$ the error rate of the test, where the error rate of the test (on a day) is the ratio of the number of positive tests to the number of total tests performed. It is observed from the plot that the (almost) unbiased RST algorithm gives the best fit to the ground truth evolution. The more aggressive (biased) CT and LBT strategies tend to overestimate the ground truth during the initial stages of the epidemic. We do not consider the problem of forming a more precise estimate using these strategies-it will require a precise knowledge of sampling probabilities and it is unclear if that will be available in practice.

\subsection{Visualization Through Geo Plots}

We depict an example spatio-temporal evolution of ground truth COVID-19 cases across the city, along with the corresponding positive cases detected in the recent past, in Fig. 12. This illustrates the spatio-temporal nature of our simulation model and can be useful for making qualitative inferences about certain testing and/or intervention strategies. It can be noted that while all tests return a good signature of the source of infection initially, LBT yields vey high number of positive tests during the initial period, making it a suitable testing strategy for early detection and containment of the spread.

\section{Effect on Interventions}

In this section, we compare the efficacy of our three testing policies in enabling interventions. As outlined earlier, an intervention policy takes into account the number of positive tests produced by the testing policy. An intervention such as lockdown is enabled once a particular trend is detected. Note that the purpose of such a lockdown is to "flatten the curve" by reducing the maximum number of daily COVID-19 cases in 


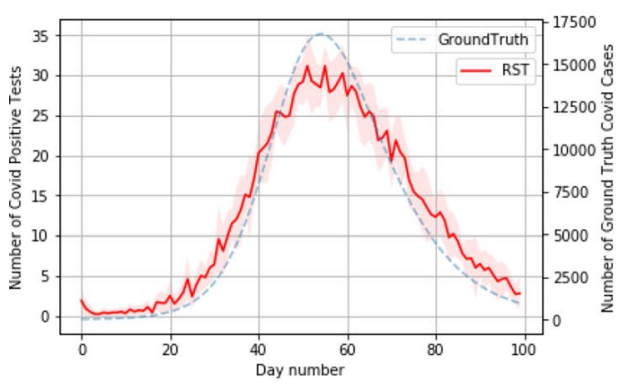

(a) Daily number of tests.

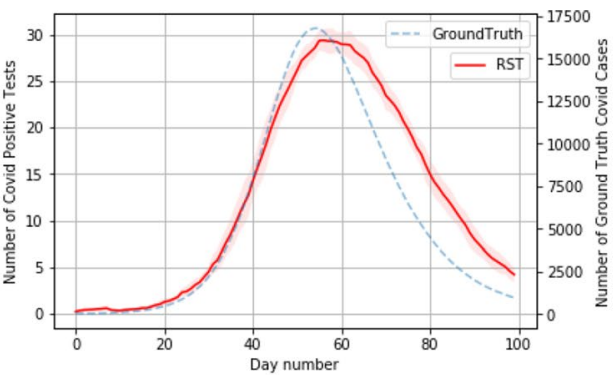

(b) Daily number of tests smoothened by taking an average over a window of 8 days.

Figure 13: Evolution of COVID-19 cases under quarantine intervention using RST

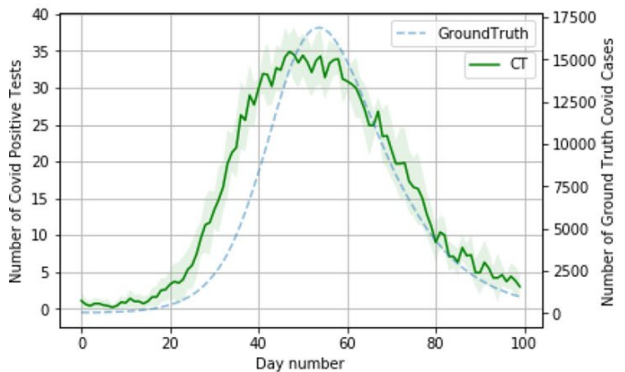

(a) Daily number of tests.

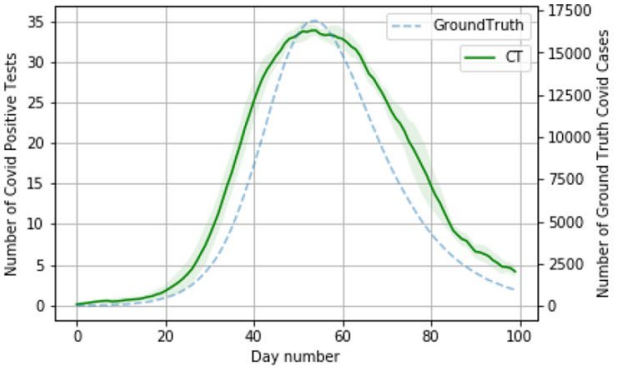

(b) Daily number of tests smoothened by taking an average over a window of 8 days.

Figure 14: Evolution of COVID-19 cases under quarantine intervention using CT.

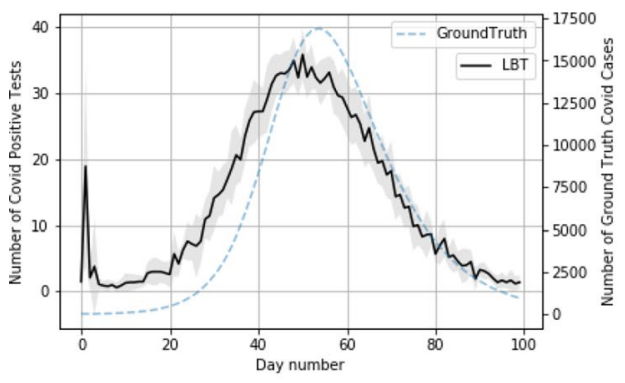

(a) Daily number of tests.

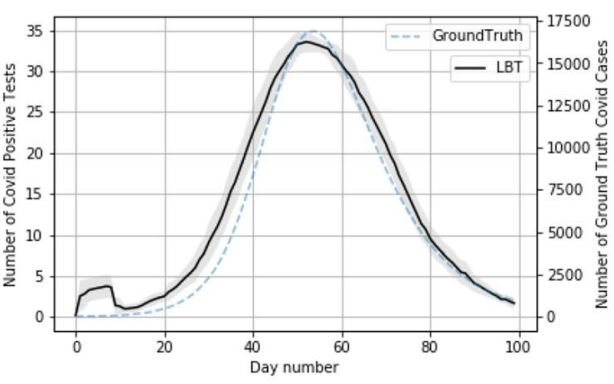

(b) Daily number of tests smoothened by taking an average over a window of 8 days.

Figure 15: Evolution of COVID-19 cases under quarantine intervention using LBT.

the ground truth. Such a reduction will reduce the stress on hospitals and other medical facilities.

We simulated COVID-19 evolution using the same rule for engaging an intervention policy using our testing polcies of RST, CT, and LBT. All the results we present in this section represent average over 10 runs of simulation; the mean behavior is depicted prominently and the spread up to standard deviation is shown in a lighter color. Briefly, we find that CT outperforms RST significantly in reducing the maximum number daily of COVID-19 cases (in the ground truth). Interestingly, LBT performs comparably with CT-recall that the former is less operation intensive than the latter. We present the three interventions we have considered in separate sections below. 


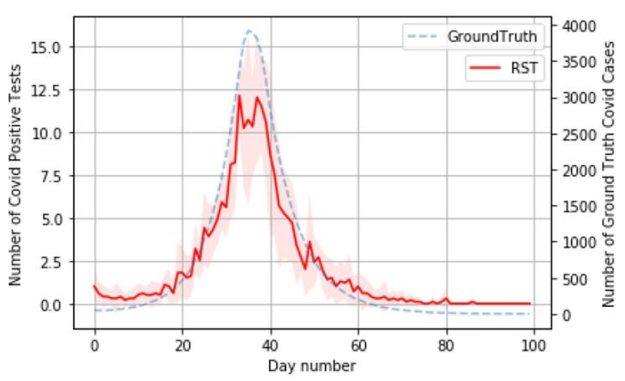

(a) Daily number of tests.

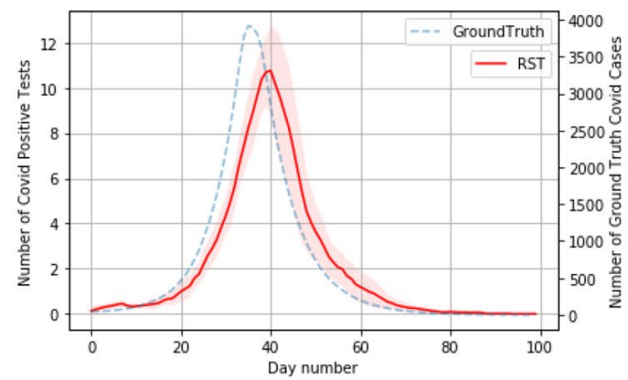

(b) Daily number of tests smoothened by taking an average over a window of 8 days.

Figure 16: Evolution of COVID-19 cases under the indefinite lockdown intervention using the thresholded smoothened slope policy for Randomized Symptomatic Testing.

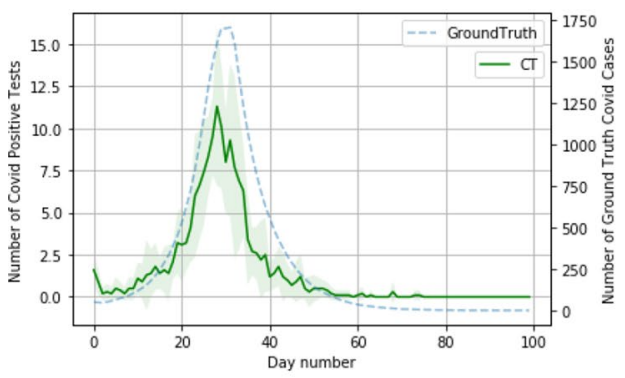

(a) Daily number of tests.

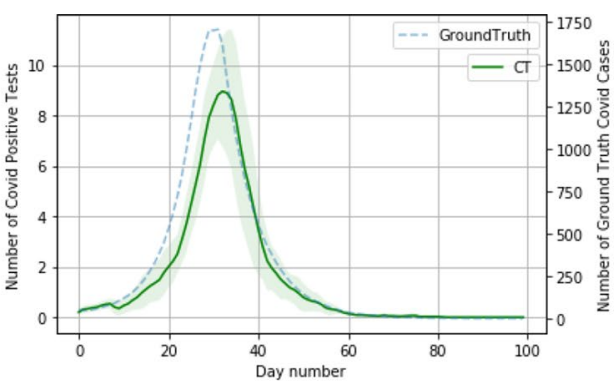

(b) Daily number of tests smoothened by taking an average over a window of 8 days.

Figure 17: Evolution of COVID-19 cases under the indefinite lockdown intervention using the thresholded smoothened slope policy for Contact Tracing.

\subsection{Quarantine}

We consider quarantine intervention where any person tested positive and all its contacts are placed under quarantine for 10 days. Note that this period of 10 days is shorted than the usual 14-21 days quarantine imposed in India; the reduction is to compensate for the absence of the $E$ state in our simplified SIR model (average $E$ to $I$ period observed is 5 days). We have considered 50 tests ( 1 test per 2000 agents) per day starting with a clustered seeding where all the initial infections of COVID-19 are placed in one locality. Specifically, we place 50 agents in COVID state I in ward number 120 (the 'Cottonpete ward'), as in the previous section. We present our results using RST, CT, and LBT in Figs. 13, 14, and 15, respectiv ely.

We observe that since the number of tests is very few, quarantine alone does not significantly reduce the maximum daily number COVID-19 cases (in the ground truth). Also, the number of positive tests that result using CT exceeds both RST and LBT, while RST captures the derivative of the ground truth COVID-19 cases well. These observations are similar to those for the case when no intervention was done and can be attributed to the unbiasedness of RST estimates when all symptomatic patients come out for testing.

\section{Remark 1}

Note that LBT gets high number of positive tests in the beginning. This phenomenon was seen for clustered seed in the previous section as well and can be attributed to the fact that LBT tests many symptomatic agents from the location where infection was initially seeded. Later, as the infection spreads 


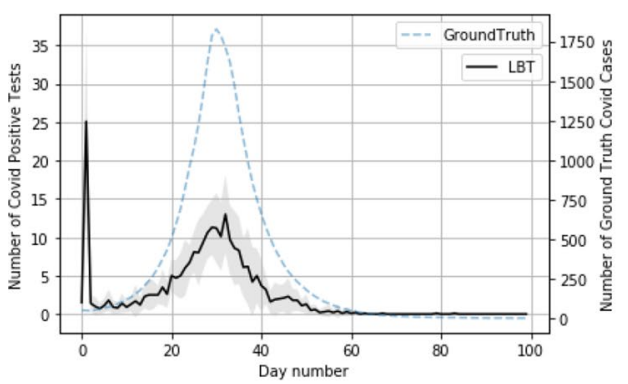

(a) Daily number of tests.

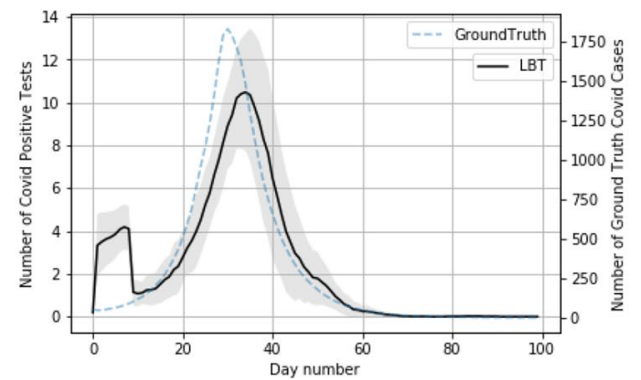

(b) Daily number of tests smoothened by taking an average over a window of 8 days.

Figure 18: Evolution of COVID-19 cases under the indefinite lockdown intervention using the thresholded smoothened slope policy for Location Based Testing.

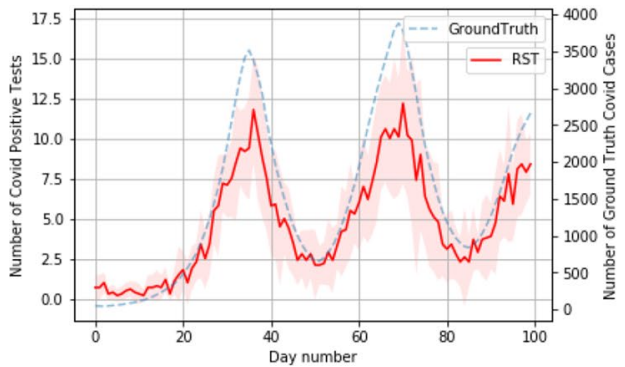

(a) Daily number of tests.

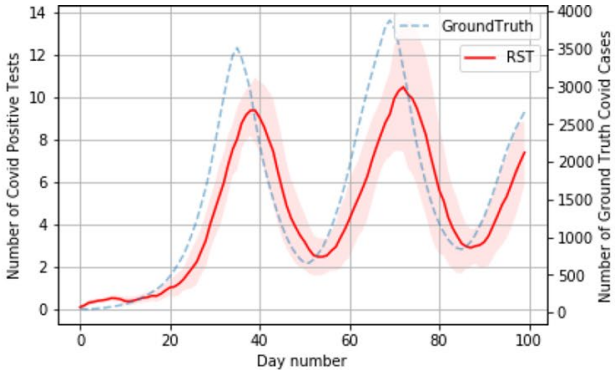

(b) Daily number of tests smoothened by taking an average over a window of 8 days.

Figure 19: Evolution of COVID-19 cases under the fixed-duration lockdown intervention using the thresholded smoothened slope policy for Randomized Symptomatic Testing.

across the city and LBT has captured a large fraction of the initial 50 cases in ward number 120, LBT starts performing comparably with CT. In fact, this observation will hold in all the other interventions as well. This feature of LBT can be exploited to enforce a local lockdown of ward number 120 early on, preventing the further spread of COVID19. However, we do not consider this intervention in this paper and will visit it in follow-up work.

\subsection{Indefinite Lockdown}

We have implemented a threshold-based lockdown policy which disables the interaction of the agents with each other once the slope of the number of positive tests graph crosses a threshold. Specifically, denoting by $P(t)$ the number of positive test outcomes, our policy computes the slope of the " 10 day chord" of a smoothened version $\bar{P}(t):=\frac{1}{8} \sum_{i=0}^{7} P(t-i)$ of $P(t)$ given by

$$
\theta(t)=\frac{\bar{P}(t)-\bar{P}(t-10)}{10} ;
$$

and starts a lockdown once $\theta(t)$ crosses a fixed threshold $\tau=0.5$. The selection of slope of the smoothened graph of $P(t)$ as a feature to use is based on our empirical observation that this slope captures the slope of the ground truth COVID-19 cases graph well. We term this policy the thresholded smoothened slope policy for lockdown. We use the same clustered seed as in the previous section and present our results using RST, CT, and LBT in Figs.16, 17, and 18, respectively.

Looking at the ground truth number of cases under three testing policies, we observe that CT reduces the maximum daily COVID-19 cases the most by about $90 \%$ of the peak value in absence of any intervention. Even RST offers a significant reduction, by about $80 \%$, but is much worse than CT. Remarkably, LBT, too offers very similar reduction as $\mathrm{CT}$ while requiring much less operational effort. 


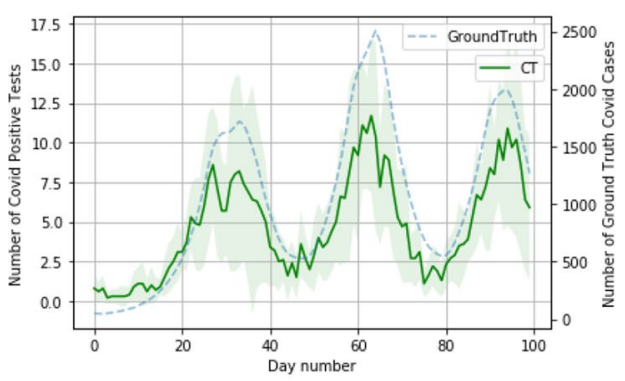

(a) Daily number of tests.

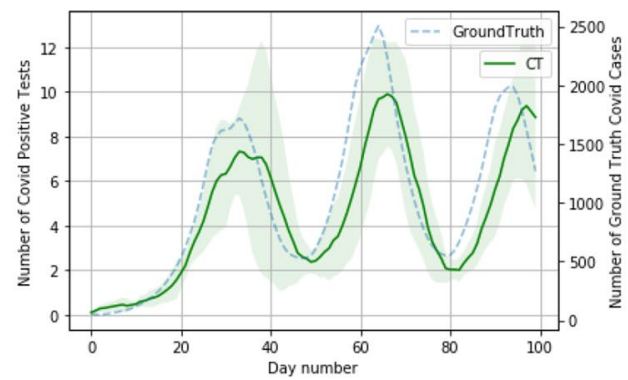

(b) Daily number of tests smoothened by taking an average over a window of 8 days.

Figure 20: Evolution of COVID-19 cases under the fixed-duration lockdown intervention using the thresholded smoothened slope policy for Contact Tracing.

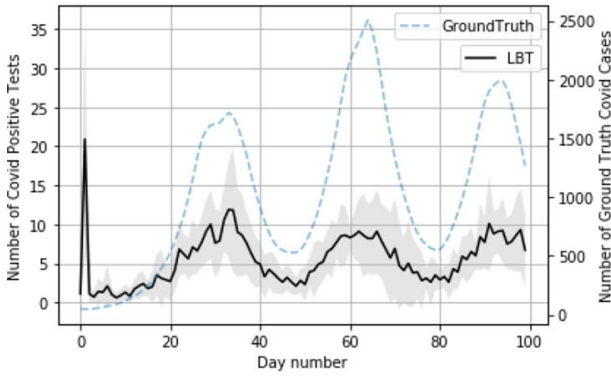

(a) Daily number of tests.

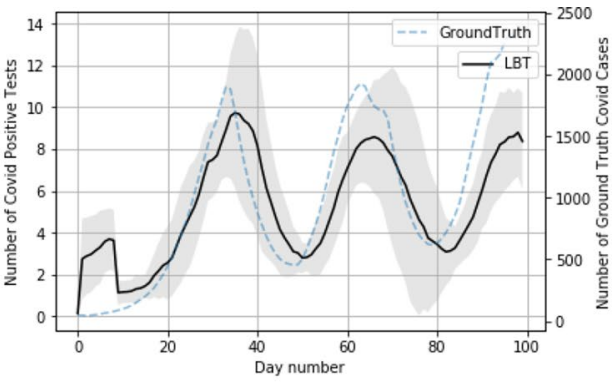

(b) Daily number of tests smoothened by taking an average over a window of 8 days.

Figure 21: Evolution of COVID-19 cases under the fixed-duration lockdown intervention using the thresholded smoothened slope policy for Location-Based Testing.

\subsection{Fixed-Duration Lockdown}

The last intervention we consider is a fixed-duration lockdown which, too, uses the thresholded smoothened slope policy to engage a lockdown. But after a lockdown is initiated, it is lifted after 14 days. We use the same clustered seed as in the previous sections and present our results using RST, CT, and LBT in Figs. 19, 20, and 21, respectively.

We observe that each testing policy results in three such lockdowns over a hundred-day period in our simulation. Interestingly, even with significantly fewer days of lockdown, the reduction in the number of ground truth COVID-19 cases is only marginally less than indefinite lockdown of the previous section. Further, here, too, CT outperforms RST significantly and LBT yields similar performance as CT.

\section{Discussion}

The design and analysis of strategies for testing a population, given limited testing resources, is a rich space of challenging problems relevant to public health policy. We have only scratched the surface by exploring how certain strategies like random sampling and contact tracing and variants inform us about the ground truth state, and what their qualitative characteristics are. We present our concluding thoughts and perspective below.

The ground truth trend signal can reveal itself even at low testing rates. Using our simulation framework with 1 test per 2000 people, we have observed that the simplest testing strategy RST captures the trend well. Note that, at the time of writing, the actual number of daily tests being conducted in India-about 1 test per 1000 people ${ }^{2}$-is almost twice this testing rate. Other strategies such as CT and our proposed LBT yield higher number of positive tests than RST, and are seen to be more effective in enabling 


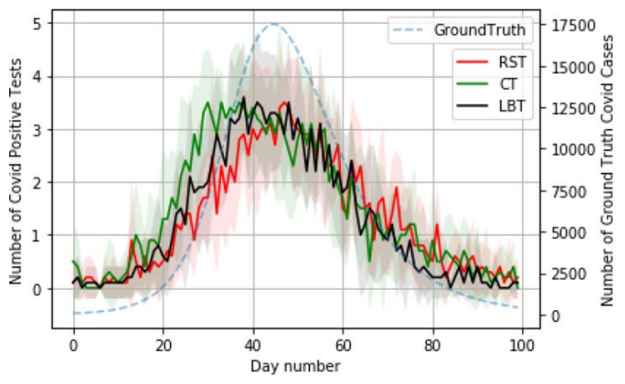

(a) Daily number of tests.

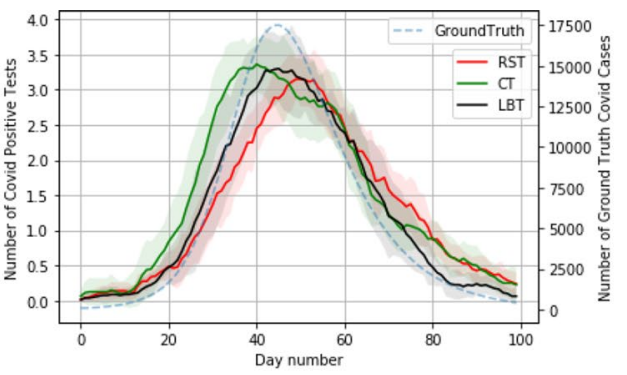

(b) Daily number of tests smoothened by taking an average over a window of 8 days.

Figure 22: Comparative test performance with uniform seeding and without intervention, for a time period of 100 days and with a testing budget of 5 tests/day. Results are averaged across 10 runs and error bars represent 1 standard deviation.

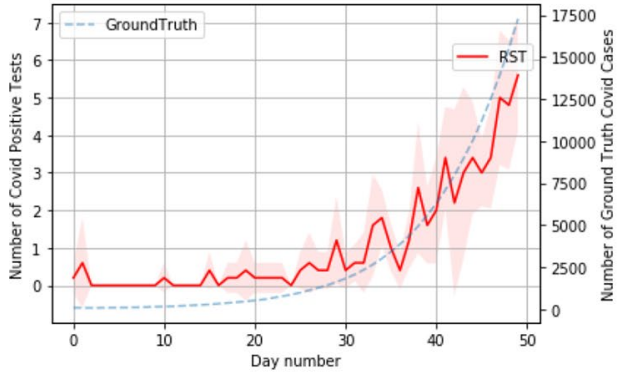

(a) Daily number of tests.

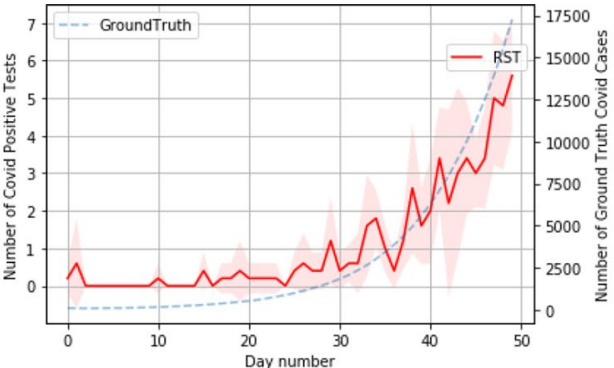

(b) Daily number of tests smoothened by taking an average over a window of 8 days.

Figure 23: Performance of RST with uniform seeding and without intervention for a population of 1 Million, for a time period of 50 days, and with a testing budget of 50 tests/day. Results are averaged across 5 runs and error bars represent 1 standard deviation.

interventions. Even at this low number of tests, positive tests reflect the ground truth trend in our experiments. We in fact even conducted experiments with uniform seeding with 5 tests per day (for a $100 \mathrm{~K}$ population; this amounts to 1 test per 20,000 people) and a shorter experiment with RST alone with 50 tests per day for a $1 \mathrm{M}$ population, whose results are displayed in Figs. 22 and 23.

Ramping up in testing capacity and different testing modalities. We have not explicitly accounted, in our modeling, for the fact that the number of tests deployed by a state or administrative unit itself changes with time. As an example, the daily number of tests conducted in Karnataka state have roughly seen a hundredfold increase, from about 600 in early April to about $60 \mathrm{~K}$ in late

\footnotetext{
${ }^{7}$ The general consensus among epidemiologists seems to be that the TPR must be kept in the range 5-10\% ('not too high, not too low').
}

August ${ }^{2}$. Along the way, we have also witnessed the rise of cheaper, faster but less accurate testing methodologies (compared to the standard RT-PCR test) such as the Rapid Antigen Test $(\mathrm{RAT})^{24}$. In addition there are antibody assays or serological tests that have been developed for post hoc identification of individuals who have been infected with the novel coronavirus, albeit with a delay ${ }^{28}$. How to most effectively deploy a battery of such testing modalities in a population, considering their accuracy (false positive/negative rates) and delayed response characteristics, remains an important unsolved problem.

Test positivity rates and what they can signify. Much of the modeling and simulation work for this article was carried out during the early stages of the pandemic in India, before rising test positivity rates (TPR) became a cause for concern and prompted the World Health Organization to issue guidelines to countries to keep TPRs low ${ }^{7}$ by expanding testing capacity ${ }^{26}$. The rationale 
behind this prescription seems to be rooted in the principle that to successfully contain an epidemic, every infected individual must ideally be tested and isolated; thus, a high TPR may signal that only a small number of tests are being used to find infected individuals where they are most likely to be found, i.e., via contact tracing or in the 'neighborhood' of already-identified positive cases, and that testing capacity ought to be ramped up. Higher TPRs indeed show up in our experiments with a very scarce test budget (approximately 60-70\% TPR at peak no. of active cases), and if one could hypothetically test the entire population at peak, then the TPR would be much lower at about $17 \%$.

It is our understanding that this paper's main goal is somewhat complementary to the question of how to control TPRs, in the sense that the former is an 'inner loop' problem of how to best use (limited) testing resources among a population, via sampling strategies, to achieve objectives (such as estimation, containment or discovery). The latter issue, however, is an 'outer loop' concern about how to change or modulate the amount of testing resources given a reasonable testing strategy (such as contact tracing) in the inner loop, based on the TPR signal, to achieve an end-to-end containment goal, and is, thus, a slower time-scale and larger scale problem of when and how to ramp up test capacity.

Other remarks. There are a few other cautionary remarks we must make: First, the sampling probabilities that emerge during test prescription by doctors in practice are difficult to evaluate and may deviate significantly from our ideal assumptions. Second, our policies rely on symptomatic patients coming out for testing (CT does not assume that). While this can be ensured by an active information campaign and ready access to doctors and digital medical advice, it is important to understand what can happen when a subsection of population is unable to report symptoms. Third, having more positive tests can perhaps offer robustness to deviations from our model in practice. Finally, we remark that we have not studied more localized interventions (though our simulations suggest that LBT will be more effective to enable a local lockdown). The effect of under reporting needs to be studied more thoroughly, too. It is of interest also to study extensive testing in an emerging hot-spot as well as pooling for more efficient testing. We plan to consider these issues in later versions of this ongoing work.

\section{Publisher's Note}

Springer Nature remains neutral with regard to jurisdictional claims in published maps and institutional affiliations.

\section{Acknowledgements}

We thank Rajesh Sundaresan for useful discussions and encouragement to build a simulation framework for testing COVID-19, Abdul Pinjari (IISc) for sharing Bengaluru traffic flow data available at CiSTUP, IISc, and Ph.D. students Debangshu Banerjee, Sayak Ray Chowdhury, Lekshmi Ramesh, and K. R. Sahasranand for help with data handling. We further thank Bharadwaj Amrutur and Hephsiba Rani, who is the officer in-change of the COVID war room of Bengaluru city, for several useful discussions related to COVID management policies. We are grateful to the anonymous referees for their useful feedback which has helped to improve the presentation of the paper.

Received: 24 August 2020 Accepted: 22 September 2020 Published online: 23 October 2020

\section{References}

1. Agrawal VS, Bhandari S, Bhattacharjee A, Deo A, Dixit N, Harsha P, Juneja S, Kesarwani P, Swamy AK, Patil P, Rathod N, Saptharishi RAYS, Sriram S, Srivastava P, Sundaresan R, Koshy N (2020) Covid-19 epidemic: Unlocking the lockdown in India, Tech. Rep

2. [Online]. Available. https://www.covid19india.org. Accessed Sept 2020

3. [Online]. Available. http://projects.datameet.org/Munic ipal_Spatial_Data/bangalore. Accessed Sept 2020

4. [Online]. Available. https://smartcities.data.gov.in. Accessed Sept 2020

5. Biswas A, Bannur S, Jain P, Merugu S (2020) COVID-19: strategies for allocation of test kits. arXiv:2004.01740

6. Danon L, Ford A, House T, Jewell C, Keeling M, Roberts G, Ross J, Vernon M (2011) Networks and the epidemiology of infectious disease. Interdiscip Perspect Infect Dis. https://doi.org/10.1155/2011/284909

7. Deshpande Y, Hosoi P, Tegling E (2020) Are the coronavirus case counts useful?. [Online]. https://idss.mit. edu/vignette/are-the-coronavirus-case-counts-useful. Accessed Sept 2020

8. Ferguson N, Cummings D, Fraser C et al (2006) Strategies for mitigating an influenza pandemic. Nature 442:448-452

9. Ferguson NM, Laydon D et al (2020) Impact of nonpharmaceutical interventions (NPIs) to reduce COVID19 mortality and healthcare demand. Imperial College COVID-19 Response Team, London 
10. Ferretti L, Wymant C, Kendall M, Zhao L, Nurtay A, Abeler-Dörner L, Parker M, Bonsall D, Fraser C (2020) Quantifying SARS-CoV-2 transmission suggests epidemic control with digital contact tracing. Science 368(6491)

11. Gopalan A, Tyagi H (2020) An agent based simulator for COVID-19 testing and policy interventions. [Online]. https://github.com/htyagi/CovidSim/. Accessed Sept 2020

12. He X, Lau E, Wu P et al (2020) Temporal dynamics in viral shedding and transmissibility of COVID-19. Nat Med 26:672-675

13. Keeling MJ, Eames K (2005) Networks and epidemic models. J R Soc Interface 2(4):295-307

14. Klepac P, Kucharski AJ, Conlan AJ, Kissler S, Tang M, Fry H, Gog JR (2020)Contacts in context: large-scale settingspecific social mixing matrices from the BBC Pandemic project. medRxiv

15. Li R, Pei S, Chen B, Song Y, Zhang T, Yang W, Shaman J (2020) Substantial undocumented infection facilitates the rapid dissemination of novel coronavirus (SARS-CoV2). Science 368:489-493

16. Linton N, Kobayashi T, Yang Y, Hayashi K, Akhmetzhanov A, Jung S-M, Yuan B, Kinoshita R, Nishiura H (2020) Incubation period and other epidemiological characteristics of 2019 novel coronavirus infections with right truncation: a statistical analysis of publicly available case data. J Clin Med 9(2):538

17. Lorch L, Trouleau W, Tsirtsis S, Szanto A, Schölkopf B, Gomez-Rodriguez M (2020) A spatiotemporal epidemic model to quantify the effects of contact tracing, testing, and containment. arXiv:2004.07641

18. M. of Health \& Family Welfare Govt. of India, Containment plan for large outbreaks, novel coronavirus disease 2019, April 2020, MOHFW [Online; posted April-2020]

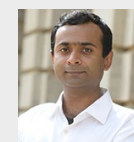

Aditya Gopalan is an Associate Professor at the Indian Institute of Science, Department of Electrical Communication Engineering. He received the Ph.D. degree in electrical engineering from The University of Texas at Austin, and was a postdoctoral fellow at the Technion-Israel Institute of Technology. His research interests include machine learning and statistical inference, control, and network algorithms.

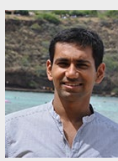

Himanshu Tyagi received the B.Tech. degree in electrical engineering and the M.Tech. degree in communication and information technology, both from the Indian Institute of Technology, Delhi, India
19. Prahladh Harsha V, Juneja S, Patil P, Rathod N, Saptharishi R, Sarath AY, Sriram S, Srivastava P, Sundaresan R, Vaidhiyan NK (2020) "Covid-19 epidemic study ii: Phased emergence from the lockdown in mumbai," Tech. Rep. [Online]. https://cps.iisc.ac.in/wp-content/ uploads/2020/08/COVID-19-Epidemic-Study-II-Phase d-Emergence-from-the-lockdown-in-Mumbai.pdf

20. PTI, India not out of woods; needs to exponentially ramp up tests: experts on COVID-19 pandemic, 6 April 2020, The Economic Times [Online; posted 6-April-2020]

21. Saikia A (2020) Testing is key to fighting coronavirus-so why does India have such a low testing rate? 27 March, Scroll.in [Online; posted 27-March-2020]

22. Shekatkar S, Pujari B, Arjunwadkar M, Hazra DK, Chaudhuri P, Sinha S, Menon GI, Sharma A, Guttal V (2020) INDSCI-SIM: a state-level epidemiological model for India. Ongoing study at https://indscicov.in/indsc isim. Accessed Sept 2020

23. Singh R, Adhikari R (2020) Age-structured impact of social distancing on the COVID-19 epidemic in India. arXiv:2003.12055

24. Tang Y-W, Schmitz JE, Persing DH, Stratton CW (2020) Laboratory diagnosis of covid-19: current issues and challenges. J Clin Microbiol 58(6):e00512-e00520

25. Thompson SK (1990) Adaptive cluster sampling. J Am Stat Assoc 85(412):1050-1059

26. W. H. Organization, "Covid-19 - virtual press conference-30 march 2020," March 2020, WHO [Online; posted March-2020]

27. Wikramaratna P, Paton RS, Ghafari M, Lourenco J (2020) Estimating false-negative detection rate of SARS-CoV-2 by RT-PCR, medRxiv

28. Winter AK, Hegde ST (2020) The important role of serology for COVID-19 control. Lancet Infect Dis 20(7):758-759

in 2007. He received the Ph.D. degree from the University of Maryland, College Park in 2013. From 2013 to 2014, he was a postdoctoral researcher at the Information Theory and Applications (ITA) Center, University of California, San Diego. Since January 2015, he has been a faculty member at the Department of Electrical Communication Engineering, Indian Institute of Science in Bangalore. His research interests broadly lie in information theory and its application in cryptography, statistics and computer science. Also, he is interested in communication and automation for city-scale systems. 